\title{
INCIDENCE OF ALVEOLAR OSTEITIS IN FEMALE PATIENTS ON ORAL CONTRACEPTIVE DRUGS UNDERGOING MANDIBULAR THIRD MOLAR SURGERY
}

\author{
Kumar Nilesh', Eesha Pisal' \\ 'Oral and Maxillofacial Surgery, School of Dental Sciences, Krishna Institute of Medical Sciences Deemed University, Karad, India \\ ${ }^{2} S$ chool of Dental Sciences, Krishna Institute of Medical Sciences Deemed University, Karad, India
}

\begin{abstract}
INTRODUCTION: Alveolar osteitis is a common complication after tooth extraction. Risk of alveolar osteitis is relatively higher following surgical removal of impacted mandibular third molars, as compared to normal extraction. ОвјестіVEs: This study was conducted to evaluate the incidence of alveolar osteitis in females taking oral contraceptive drugs.

MATERIAL AND METHODS: This retrospective study evaluated the incidence of alveolar osteitis in patients after surgical removal of mandibular $3^{\text {rd }}$ molar. Patient records were evaluated for demographic details, history of procedures, intake of oral contraceptive drugs during extraction, and complications. Data obtained from the retrospective analysis was divided into male, females on contraceptives, and females not on contraceptive drugs. The incidence of alveolar osteitis was evaluated in these three groups.

RESULTS: Data of 660 patients who had undergone $3^{\text {rd }}$ molar surgery was evaluated, out of which 452 were male and 208 were female patients. Total of 42 female patients were on oral contraceptive drugs. 51 patients (7.72\%) had alveolar osteitis on post-operative follow-up. Out of these 51 patients, 31 were males and 20 were females. Incidence of alveolar osteitis in females on oral contraceptive drugs was almost double as compared to females without contraceptive drugs and males.

Conclusions: This study supported the hypothesis that oral contraceptive drug increases the risk of alveolar osteitis. However, our study was a retrospective analysis, therefore has certain limitations. A prospective research with grading of surgical difficulty could help to standardize these results.
\end{abstract}

KEY wORDs: dry socket, surgical extraction, impaction, complication, wisdom teeth, infection.

J Stoma 2019; 72, 6: 252-255

DOI: https://doi.org/10.5114/jos.2019.93844

\section{INTRODUCTION}

Tooth extraction is one of the most commonly performed procedures in dental clinic and may be associated with a variety of complications. The severity and frequency of these complications differ depending on type of extraction. A simple non-surgical (intra-alveolar) extraction is associated with far lower risk of complication, as compared to surgical or trans-alveolar extraction. Surgical extraction of impacted mandibular $3^{\text {rd }}$ molar is related with common post-operative sequelae like pain, discomfort, swelling, and trismus as well as certain undesirable complications. The commonly reported complications include: tooth/ root displacement, alveolar bone

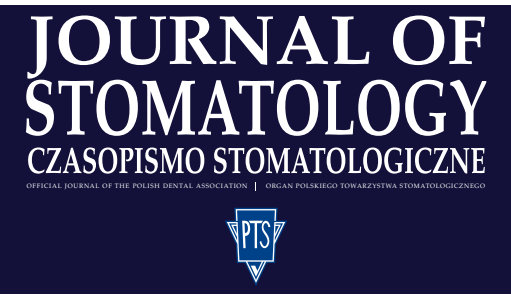

AdDRESS FOR CORRESPONDENCE: Dr. Kumar Nilesh, Oral and Maxillofacial Surgery, School of Dental Sciences, Krishna Institute of Medical Sciences Deemed University, Malkapur, 415110, Karad, India, e-mail: drkumarnilesh@yahoo.com

RECEIVED: 20.10.2019 • ACCEPTED: 19.02.2020 • PUBLISHED: 10.03.2020 
fracture, excessive bleeding and pain, root breakage, jaw fracture, trismus, alveolar osteitis, and inferior/lingual nerve paresthesia [1].

Alveolar osteitis (AO) is a complication arising due to dislodgment of blood clot from the extraction socket, leading to hampered healing at the extraction site. It usually presents as pain at the site of extraction, non-healing extraction socket, halitosis, and restricted mouth opening, resulting in significant discomfort and compromised quality of life. The reported incidence of AO varies between 1 and $4 \%$ after routine extraction [2]. However, the risk of $\mathrm{AO}$ after surgical extraction of impacted mandibular $3^{\text {rd }}$ molar is significantly higher, resulting in about 5-30\% [3]. Various risk factors are known to affect the incidence of $\mathrm{AO}$ including patient's age, gender, surgical trauma and difficulty, surgeon's experience, compliance to post-operative instruction, post-extraction socket irrigation, smoking, and the use of oral contraceptives. Oral contraceptive (OC) drugs are known to enhance the fibrinolysis of blood clot within extraction socket, resulting in increased risk of developing AO.

\section{OBJECTIVES}

The present study was designed to evaluate the effect of oral contraceptive pills on the incidence of $\mathrm{AO}$ after $3^{\text {rd }}$ molar surgery. The hypothesis at the beginning of the data analysis: the incidence of $\mathrm{AO}$ would be higher in females taking OC drugs during surgical procedure as compared to females not on OCs and males patients. Other complications associated with $3^{\text {rd }}$ molar surgery were also studied.

\section{MATERIAL AND METHODS}

A retrospective analysis of patients who underwent surgical removal of mandibular $3^{\text {rd }}$ molar under local anesthesia from January 2017 to February 2019 was carried out. Data analysis were derived from patients' records and included demographic details, procedures performed, history of $\mathrm{OC}$ in female patients, postoperative follow-up of complications and their management. Patients with incomplete records and those who underwent surgery for soft tissue impaction or completely bony impacted mandibular $3^{\text {rd }}$ molars were excluded from the study. Impacted $3^{\text {rd }}$ molars, which did not require bone removal and tooth sectioning, and patients who were smokers were additionally excluded from the study. Cases of partial bony impacted $3^{\text {rd }}$ molars of varying degree of difficulty and those performed by a single surgeon were included in the study. All patients received the same post-operative care as per departments' protocol. Patient who reported with pain and presence of greyish slough or lack of clot in extraction socket along with tenderness on probing at the postoperative follow-up were considered positive for AO. Treatment notes indicating management given for $\mathrm{AO}$ were also considered positive for AO. Data was analyzed for gender and age distribution, the incidence of $\mathrm{AO}$ in male and female groups as well as group of patients using OC drugs. The data was evaluated using SPSS (Statistical Package for the Social Sciences). Other complications observed after mandibular $3^{\text {rd }}$ molar surgery was also investigated.

\section{RESULTS}

The 683 patients who underwent $3^{\text {rd }}$ molar surgery from January 2017 to February 2019 were screened for availability of complete details required for data analysis. Total of 660 cases fulfilling the inclusion criteria were included after the initial screening, which comprised of 452 male $(68.49 \%)$ and 208 female (31.51\%) patients, within the mean age group of 28.4 years. Out of 208, 42 female patients were on OC drugs during the surgical procedure.

Total of 51 patients $(7.72 \%)$ reported with complication of AO. Incidence of AO in female group without OC was $7.83 \%(n=13)$, whereas those on OC drugs was $16.66 \%(n=7)$. Among the male group, 31 patients reported with AO (6.85\%) (Table 1).

$\chi^{2}$ test was done to verify an association between exposures of OC drugs and $\mathrm{AO}$ in females. Occurrence of $\mathrm{AO}$ in female patients on OC drugs was 2.3 times more compared to females not on OC drugs. $\chi^{2}$ test was performed to examine an association between occurrence of $\mathrm{AO}$ in females on OC drugs and males. With odds ratio of 2.716, the occurrence of $\mathrm{AO}$ in females on OC drugs was 2.7 times more than males (95\% confidence interval [CI]:

TABLE 1. Incidence of alveolar osteitis in the studied population

\begin{tabular}{|c|c|c|c|c|c|}
\hline \multirow{2}{*}{ Population } & \multicolumn{2}{|c|}{ AO present } & \multicolumn{2}{|c|}{ AO absent } & \multirow{2}{*}{ Total } \\
\hline & $n$ & $(\%)$ & $n$ & $(\%)$ & \\
\hline Females exposed to $0 \mathrm{C}$ drugs & 7 & 16.7 & 35 & 83.3 & 42 \\
\hline Females not exposed to OC drugs & 13 & 7.8 & 153 & 92.2 & 166 \\
\hline Males (not exposed to OC drugs) & 31 & 6.9 & 421 & 93.1 & 452 \\
\hline Total & 51 & 7.7 & 609 & 92.3 & 660 \\
\hline
\end{tabular}

$A O$ - alveolar osteitis, OC - oral contraceptive 
1.12-6.61). There was a significant association between both the groups $\left(\chi^{2}=5.21\right.$, $p$ value $\left.=0.02\right)$.

Besides AO, other complications associated with mandibular $3^{\text {rd }}$ molar surgery were studied. For descriptive purpose, these complications were grouped into intra-operative and post-operative complications. The intra-operative complications included root fracture $(17.72 \%, n=117)$ and damage/luxation of adjacent tooth $(1.81 \%, n=12)$. Other intra-operative complications like tooth/root displacement into facial space, excessive bleeding, and jaw fracture were not reported. Post-operative complications included swelling in $43 \%$ $(n=284)$, trismus in $34.4 \%(n=227)$, inferior alveolar or lingual nerve paraesthesia in $1.5 \%(n=10)$, postoperative bleeding in $0.4 \%(n=3)$, and infection (dentoalveolar and/or facial space infection) in $5.3 \%(n=35)$ of patients.

\section{DISCUSSION}

Alveolar osteitis is one of the known complications of tooth extraction. Crawford in 1896, coined the term "dry socket" indicating loss of blood clot, which results in AO [2]. Since then, "dry socket" has been given several names including fibrinolytic alveolitis, localized osteomyelitis, and post-operative alveolitis [4]. It always presents as pain on $1^{\text {st }}$ to $3^{\text {rd }}$ day after extraction, with evidence of completely or partially dislodged blood clot from the extraction socket revealing bare bone. Other features associated with AO include halitosis, trismus, cervical lymphadenopathy, swelling, cellulitis, and tenderness on probing [5].

Since the use of OC drugs from the 1960's, there has been a documented rise in prevalence of $\mathrm{AO}$ in females. This was supported by Field et al. who observed no significant difference in incidence of AO between both sexes in 1971; however, the same author in 1983, reported significantly higher incidence of $\mathrm{AO}$ in females, which was possibly due to increased use of OC during that period [6]. OC drugs are known to modify coagulation and fibrinolytic activity. There is an increase in activity of coagulation factors VII and X as well as fibrinogen with the use of OC, which promotes coagulation [7]. On the other hand, there is accentuation of fibrinolytic potential due to an increase in plasminogen function [8]. A balance of these two activities in favor of fibrinolysis is believed to be responsible for breakdown of clot in the extraction socket in patients on OC drugs, thus predisposing them to AO. Schow [9] and Sweet [10] supported the hypothesis, and reported higher incidence of AO in females on OC drugs at $45 \%$ and $19 \%$, respectively, as compared to $17 \%$ and $17 \%$ in females not on $\mathrm{OC}$, respectively. According to the present study, the risk of $\mathrm{AO}$ in females on OC drugs was almost double compared to females not using OC drugs and males. This finding is consistent with some of the recently reported studies $[11,12]$. Several studies have reported no statistically significant difference in incidence of $\mathrm{AO}$ among females taking and not taking OC. However, most of these studies had a small sample size [13]. The risk of AO has been reported to vary based on duration of OC therapy. Hedlin et al. stated that the fibrinolytic activity in blood circulation increased from the first day after taking OC drugs [14]. An increased risk of $\mathrm{AO}$ was seen after extraction of mandibular $3^{\text {rd }}$ molars during day 1 to 22 of the use OC drugs [15].

This study supports the hypothesis that OC increases the risk of AO. However, being a retrospective analysis, it has certain limitations. The presence of pain at extraction site with partial/complete loss of clot or presence of slough in socket was regarded as positive diagnostic tool for AO in the present study. Lack of mandatory follow-up after extraction could possibly mean that all the patients who developed AO may not have necessarily reported, thus lowering the incidence of AO. There are various other factors, which are known to influence the risk of $\mathrm{AO}$. One of the risk factors for $\mathrm{AO}$ is age of the patient. In our study, there was no statistical difference in the mean age of the 3 groups studied. As cigarette smoking is one of the known risk factors for $\mathrm{AO}$, only non-smokers were included for data analysis. An increased surgical difficulty and trauma result in increased risk of AO. To overcome this bias, conditions such as soft tissue impaction and completely bony obstructed tooth were not included in this study. This eliminated procedures, which were either too simple or too complicated. Exclusion of complete bony impacted tooth could possibly be a reason for relatively lower incidence of AO, as compared to previous studies [11]. The study included procedures, which were performed by a single surgeon, thereby eliminating bias among the three groups. A prospective study design with grading of surgical difficulty based on various rating scales can further standardize these results. There is also a need to study the possibility of variations in the risk of $\mathrm{AO}$ based on the day of OC therapy.

\section{CONCLUSIONS}

Although many complications are associated with surgical removal of impacted mandibular $3^{\text {rd }}$ molar, $\mathrm{AO}$ is one of the most common. This retrospective data analysis showed that female patients taking oral contraceptive pills and undergoing mandibular $3^{\text {rd }}$ molar surgery are at higher risk of developing $\mathrm{AO}$, as compared to male patients and female patients not using OC during the procedure. The overall incidence of $\mathrm{AO}$ was $7.72 \%$ in the study population. The incidence of alveolar osteitis in females on oral contraceptive drugs was almost double (16.66\%), as compared to females not using oral contraceptive drugs and males. 


\section{CONFLICT OF INTERESTS}

The authors declare no potential conflicts of interest with respect to the research, authorship, and/or publication of this article.

\section{References}

1. Bhujbal R, A Malik N, Kumar N, Kv S, I Parkar M, Mb J. Comparative evaluation of platelet rich plasma in socket healing and bone regeneration after surgical removal of impacted mandibular third molars. J Dent Res Dent Clin Dent Prospects 2018; 12: 153-158.

2. Noroozi AR, Philbert RF. Modern concepts in understanding and management of the "dry socket" syndrome: comprehensive review of the literature. Oral Surg Oral Med Oral Pathol Oral Radiol Endod 2009; 107: 30-36.

3. Eshghpour M, Rezaei NM, Nejat A. Effect of menstrual cycle on frequency of alveolar osteitis in women undergoing surgical removal of mandibular third molar: a single blind randomized clinical trial. J Oral Maxillofac Surg 2013; 71: 1484-1489.

4. Hermesch CB, Hilton TJ, Biesbrock AR, et al. Perioperative use of $0.12 \%$ chlorhexidine gluconate for the prevention of alveolar osteitis. Efficacy and risk factor analysis. Oral Surg Oral Med Oral Pathol Oral Radiol Endod 1998; 85: 381-387.

5. Alwraikat AA. Alveolar osteitis: incidence and risk factors following third molar surgery in Jordan. Pak Oral Dental J 2009; 29: 19-22.

6. Field EA, Speechley JA, Rotter E, Scott J. Dry socket incidence compared after a 12 year interval. Br J Oral Maxillofac Surg 1985; 23: 419-425.

7. Levi M, Middeldorp S, Büller HR. Oral contraceptives and hormonal replacement therapy cause an imbalance in coagulation and fibrinolysis which may explain the increased risk of venous thromboembolism. Cardiovasc Res 1999; 41: 21-24.

8. Norris LA, Bonnar J. Haemostatic changes and the oral contraceptive pill. Baillieres Clin Obstet Gynaecol 1997; 11: 545-564.

9. Schow SR. Evaluation of postoperative localized osteitis in mandibular third molar surgery. Oral Surg 1974; 38: 352-358.

10. Muhonen A, Venta I, Ylipaavalniemi P. Factors predisposing to postoperative complications related to wisdom tooth surgery among university students. J Am Coll Health 1997; 46: 39-42.

11. Almeida LE, Pierce S, Klar K, Sherman K. Effects of oral contraceptives on the prevalence of alveolar osteitis after mandibular third molar surgery: a retrospective study. Int J Oral Maxillofac Surg 2016; 45: 1299-1302.

12. Garcia AG, Grana PM, Sampedro FG, Diago MP, Rey JM. Does oral contraceptive use affect the incidence of complications after extraction of a mandibular third molar? Br Dent J 2003; 194: 453-455.

13. Larsen PE. Alveolar osteitis after surgical removal of impacted mandibular third molar. Identification of the patient at risk. Oral Surg Oral Med Oral Pathol 1992; 73: 393-397.

14. Hedlin AM, Monkhouse FC. Changes in spontaneous fibrinolytic activity during use of oral contraceptives. Obstet Gynecol 1971; 37: 225-229.

15. Catellani JE, Harvey S, Erickson SH, Cherkin D. Effect of oral contraceptive cycle on dry socket (localized alveolar osteitis). J Am Dent Assoc 1980; 101: 777-783. 